\title{
Spinal epidural hematoma in an infant patient: a differential diagnostic of spinal cord compression syndrome
}

\author{
Felipe Gutierrez Pineda, Mauro Marcelo Suarez Marin, Daniela Perez Cadavid
}

\author{
Department of Neurosurgery, \\ University of Antioquia, School of \\ Medicine, Medellín, Colombia \\ To whom correspondence \\ should be addressed: Felipe \\ Gutierrez Pineda, MD \\ e-mail: \\ felipegpp23@gmail.com \\ Available at: \\ http://www.archpedneurosurg.com.br/
}

\begin{abstract}
A spontaneous spinal epidural hematoma is a collection of blood in the epidural space of the spine occurring in the absence of trauma or other pathological blood conditions, this epidural blood clot has the risk to compress the spinal cord and impact negatively on the neurological outcome of the patients. With a low incidence on pediatrics this condition has not been studied deep, with difficulties on the clinical approach in infants and children due to the limitation in the pediatric neurological exam with the risk to be misdiagnosed. We describe a rare case of spinal cord compression on a young 14 months-old patient with a spontaneous cervicothoracic epidural hematoma that presented with a ascending paralysis. Magnetic resonance imaging of the spine demonstrated an extensive epidural hematoma between $\mathrm{C} 4$ to T5 with spinal cord compression. An emergency laminectomy was performed with evacuation of the epidural blood clot with a total improve of the neurologic symptoms and without any residual hematoma following 3 months of the surgery. The spontaneous spinal epidural hematoma is rare cause of spinal cord compression on infants and children with high risk of neurological impact without prompt diagnosis with MRI and emergent surgical evacuation.
\end{abstract}

Keyworks: Spontaneous spinal epidural hematoma, children, spinal cord compression, magnetic resonance

\section{INTRODUCTION}

Spontaneous spinal epidural hematoma is a rare cause of spinal cord compression during childhood. Some authors define this pathology as any blood collection in the epidural spinal space of non-traumatic origin, while others define it as any hematoma that occurs in the absence of any identifiable cause like arteriovenous malformation, bleeding diathesis and minor trauma (1).

The diagnosis of this rare disease is challenging on infants because of the unspecific symptoms at presentation and the limited neurological examination in this population (2). MRI has been proven to be the imaging of choice to diagnoses this entity with a typical appearance of the hematoma (3). Here, we present a rare case of spontaneous cervical epidural hematoma on a 14 month - old male patient that require surgery decompression and evacuation with a complete neurological improve after 3 months.

\section{CASE REPORT}

A 14 month - old male patient without any previous medical history consult to the pediatric emergency department with a $10 \mathrm{~h}$ history of neck pain and irritability associated with lower limbs weakness and fever (38.5C). The initial diagnosis of central nervous system infection was suspected and a lumbar puncture was done. Cerebrospinal 


\section{Spinal epidural hematoma in an infant patient: a differential diagnostic of spinal cord compression syndrome}

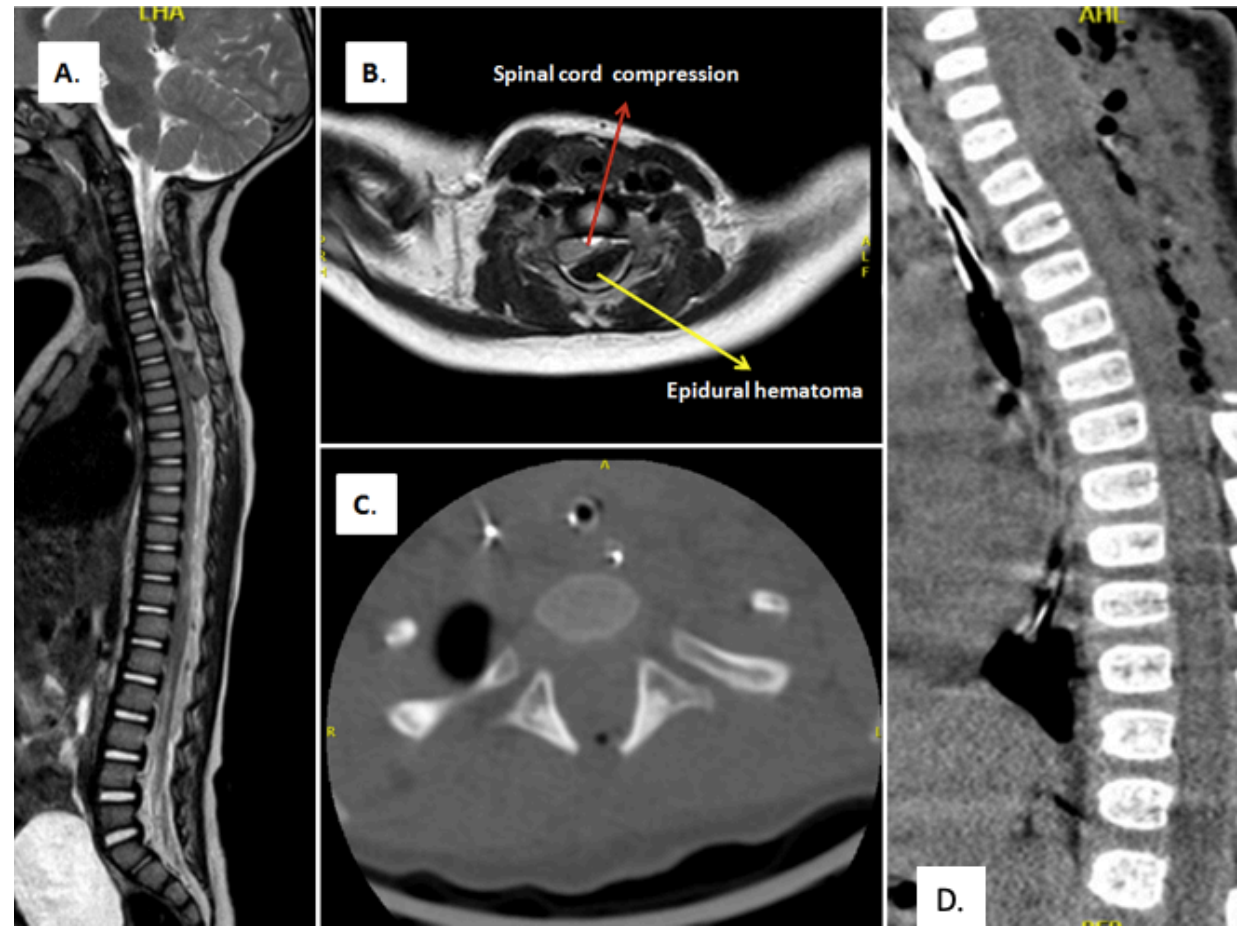

Figure 1 - Magnetic resonance image on sagittal view showing a cervicothoracic epidural hematoma from C4 to T5 on T2WI (A), MRI on axial view showing spinal cord compression (red arrow) due to epidural hematoma (B), Laminectomy extension with bilateral facet joints integrity (C), Postoperative CT scan without residual epidural hematoma and cervicothoracic laminectomy from C4 to T4 vertebral levels (D)

fluid analysis showed high protein concentration $(360 \mathrm{mg} / \mathrm{dl}$, white blood cell count of 12.5 cells $/ \mathrm{mm} 3$ with monocyte predominance, $55 \mathrm{mg} / \mathrm{dl}$ of glucose and 2.07 of lactate) intravenous antibiotic was given (vancomicyn plus ceftriaxone) and after no clinical improvement during the next 48 hours an additional diagnosis of Guillain-Barré syndrome was associated. A metilprednisolone pulse was add to the treatment without any neurological improve, following 4 days of the neurological symptoms presentation , a neurosurgery consult was performed to rule out a spinal compression syndrome.

During the following days, a neurological improvement was seen on his limbs weakness (Lower limbs 2/5 and upper limbs 4/5) with an initial postoperative CT scan without any residual epidural hematoma of the spine (Figure 1). A spinal angiogram was performed after two day from his surgery ruling out any vascular malformation from the spinal vessels. The pathology report confirmed the diagnosis of a vascular hematoma without any vascular malformation, tumor or other spinal abnormality. A normal coagulation profile was seen and any bleeding diathesis was ruled out by the hematologist group.

On the first postoperative visit to the neurosurgery department following 14 days from his surgery a gradually improve of his neurological status was seen, without any sensorimotor residual deficits. A control spinal and cerebral angiogram and a control MRI performed 3 months postoperatively showed a complete evacuation of the epidural hematoma, without any vascular malformation or spinal tumor (Figure 2), the patient was capable to walk without any neurological impairment and was discharged.

\section{DISCUSSION}

Spinal epidural hematoma without significant trauma or any pathological condition is rare, it may affect any age but its rarest in children and even more in infants, with an estimated incidence of 0,1 per 100.000, accounting for less than $1 \%$ of all epidural compression lesion of the spine (4). Literature of this disease is restricted to case reports and cases series.

Initial symptoms of spontaneous spinal epidural hematoma (SSEH) are non-specifics on infants, the majority of the cases presents with irritability and neck pain, with the neurological deficits been minimal and often no recognized until its declining (5). On previous case reports some differential diagnosis include Guillain-Barré syndrome, vascular malformation such as extradural arteriovenous fistula and infectious diseases $(3,5,6,7,8)$. 


\section{Spinal epidural hematoma in an infant patient: a differential diagnostic of spinal cord compression syndrome}
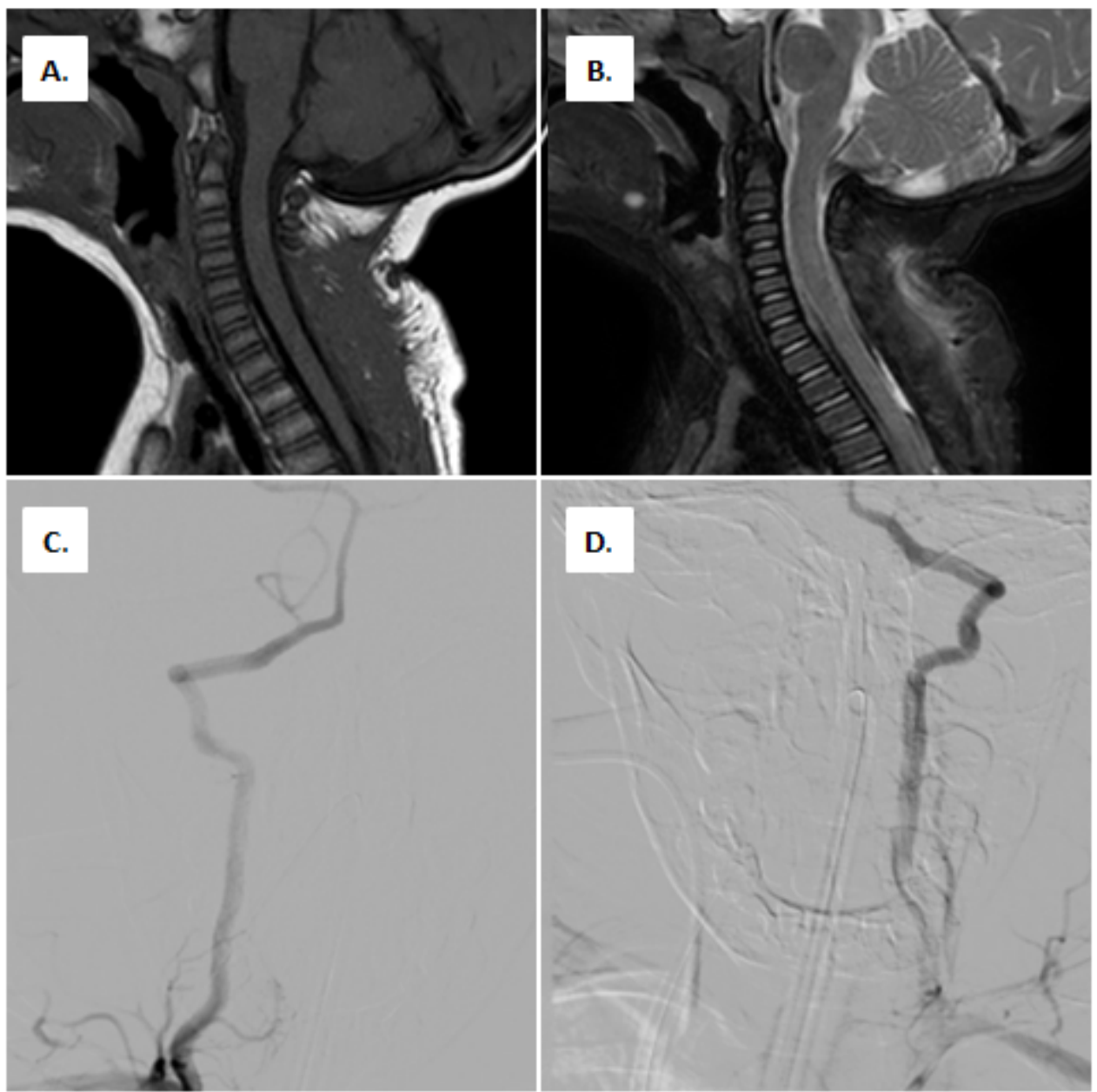

Figure 2 - Postoperative magnetic resonance image after 3 months of surgical evacuation of cervical epidural hematoma without any residual blood clot on cervicothoracic spine on T1WI -T2WI (A-B), Postoperative spinal angiography without vascular malformation after 3 months of surgical evacuation (C-D)

The pathogenesis of this disease remains unclear; most series consider a venous bleeding as the origin of the hematoma due to a rupture of a "locus minoris resistentiae" in the epidural valveless venous plexus, making the epidural space vulnerable to any intrinsic chance in pressure $(9,10)$.

Magnetic resonance imaging (MRI) is the modality imaging of choice to diagnoses spontaneous spinal epidural hematoma, been able to inform the extent, age, location and compression effects of the blood collection to the cord, T1 Weighted image helps in the follow up evaluation and the T2 Weighted images is crucial in the differentiation between epidural hematoma from a tumor $(10,11)$.If a vascular enlargement are reveled on MRI, a vascular malformation must be suspected and an spinal angiography must be done prior to any surgical intervention.
The location of the SSEH has been described more frequent on the cervico-thoracic levels, believed to be due to a rupture of the venous plexus because of the increasing abdominal or intrathoracic pressure due to straining, coughing, or sneezing, with only few cases on the lumbar spine (12).

Cord compression due to a SSEH is a critical neurosurgery emergency and surgical decompression of the spinal cord is widely accepted as the treatment of choice to prevent neurological deficits and any associated morbidity (13), treatment delay from clinical presentation to treatment has been reported to be from 2.6 -14.8 days, with a median of 5.6 days (14). 


\section{Spinal epidural hematoma in an infant patient: a differential diagnostic of spinal cord compression syndrome}

Recurrence of spontaneous epidural hematomas is rare, has been described only in case reports, giving the necessity to been alert about its risk on any hematoma without significant trauma or any prior pathological condition , unlike its has been described to occur on a short period of time after the first bleeding episode, Amoreira and Sainz has described a rare case of spontaneous spinal hematoma recurrence after two years of follow up (15), making very important that the patient and its family be informed about the risk of recurrence and the need of medical consultation if the symptoms return.

The initial neurological evaluation showed an ill, irritable patient with quadriplegia (lower limbs $1 / 5$ bilateral and upper limbs weakness $2 / 5$ bilateral), priapism and an absent sphincter tonus, deep tendon reflexes ( lower limbs +/++ and upper limbs $++/++)$, hypotension and bradycardia. Whit these clinical findings an emergent spinal MRI was done reveling a cervico-thoracic epidural hematoma with cord compression from C4 to T5 (Figure 1). The patient underwent an emergency surgery with an effective decompression ( C4 to T4) with epidural blood clot evacuation, any vascular malformation or tumor was seen on the epidural space, Histopathology samples was taken from the epidural blood collection.

\section{CONCLUSIONS}

Spontaneous spinal epidural hematomas are a rare condition on pediatrics patients, due to a high risk of neurological impairment, it must be suspected in any infant with irritability, weakness and neurological deficits of unknown origin taking on account in any child with spinal cord compression syndrome as a differential diagnosis. A prompt MRI and surgical evacuation have to be done on time to ensure a good prognosis and improve the neurological outcome

\section{DISCLOSURES}

\section{Ethical approval}

This study was performed in line with the principles of the Declaration of Helsinki. Approval was granted by the local Ethics Committee

\section{Consent to participate}

The patient gave consent to use his information and images for research proposes.

\section{Consent for publication}

The patient gave consent to use his information and images for publication.
The authors declare that they have no conflict of interest.

\section{REFERENCES}

1. Kim M, Hoffman J, Amin A, Purohit M, Mohan A. Spontaneous spinal epidural hematoma in an infant. Pediatric emergency care. 2020 Aug 1;36(8):e467-92.

2. Patel H, Boaz JC, Phillips JP, Garg BP. Spontaneous spinal epidural hematoma in children. Pediatric neurology. 1998 Oct 1;19(4):302-7.

3. Schoonjans AS, De Dooy J, Kenis S, Menovsky T, Verhulst S, Hellinckx J, Van Ingelghem I, Parizel PM, Jorens PG, Ceulemans B. Spontaneous spinal epidural hematoma in infancy: review of the literature and the "seventh" case report. european journal of paediatric neurology. 2013 Nov 1;17(6):537-42.

4. Holtås $S$, Heiling $M$, Lönntoft $M$. Spontaneous spinal epidural hematoma: findings at MR imaging and clinical correlation. Radiology. 1996 May;199(2):409-13.

5. Lee JS, Yu CY, Huang $\mathrm{KC}$, Lin $\mathrm{HW}$, Huang $\mathrm{CC}$, Chen $\mathrm{HH}$. Spontaneous spinal epidural hematoma in a 4-monthold infant. Spinal Cord. 2007 Aug;45(8):586-90.

6. Yu JX, Liu J, He C, Sun LY, Xiang SS, Ma YJ, Bian LS, Hong T, Ren J, Tao PY, Li JW. Spontaneous spinal epidural hematoma: a study of 55 cases focused on the etiology and treatment strategy. World neurosurgery. 2017 Feb 1;98:546-54.

7. Kondo A, Yamaguchi $H$, Ishida $Y$, Toyoshima D, Azumi M, Akutsu N, Koyama J, Kurosawa H, Kawamura A, Maruyama A. Spontaneous spinal epidural hematoma mimicking Guillain-Barre syndrome. Brain and Development. 2019 Apr 1;41(4):392-5.

8. Ravid S, Schneider S, Maytal J. Spontaneous spinal epidural hematoma: an uncommon presentation of a rare disease. Child's nervous system. 2002 Jul 1;18(67):345-7.

9. Groen RJ, Ponssen H. The spontaneous spinal epidural hematoma: a study of the etiology. Journal of the neurological sciences. 1990 Sep 1;98(2-3):121-38.

10. Baeesa S, Jarzem P, Mansi M, Bokhari R, Bassi $M$. Spontaneous spinal epidural hematoma: correlation of timing of surgical decompression and MRI findings with functional neurological outcome. World neurosurgery. 2019 Feb 1;122:e241-7.

11. Liao CC, Lee ST, Hsu WC, Chen LR, Lui TN, Lee SC. Experience in the surgical management of spontaneous spinal epidural hematoma. Journal of Neurosurgery: Spine. 2004 Jan 1;100(1):38-45.

12. Soltani S, Nogaro MC, Rougelot C, Newell N, Lim K, Kieser DC. Spontaneous spinal epidural haematomas in children. European Spine Journal. 2019 Oct;28(10):2229-36. 
13. Shin JJ, Kuh SU, Cho YE. Surgical management of spontaneous spinal epidural hematoma. European Spine Journal. 2006 Jun;15(6):998-1004.

14. Carlhan-Ledermann A, Laubscher B, Steinlin M, Ulrich CT, Verma RK, Rizzi M, Maduri R, Grunt S. Spinal epidural hematoma without significant trauma in children: two case reports and review of the literature. BMC pediatrics. 2020 Dec;20(1):1-7.

15. Gepp R, Quiroga MR. Recurrent spontaneous spinal epidural hematoma in children: case report. Archives of Pediatric Neurosurgery. 2019 Dec 13;1(1 (SeptemberDecember)):25-7. 